\title{
Evaluación de la función vestibular angular: Prueba de impulso cefálico multiaxial a ojo desnudo
}

\section{Angular vestibular function assessment: Multiaxial Head Impulse Test}

Hayo Breinbauer K', Karina Aracena C², José Luis Anabalón B², Sergio Aladro G³ , María Angeles Baeza A'.

\begin{abstract}
RESUMEN
Introducción: La prueba de impulso cefálico consiste en un examen rápido, sencillo para evaluar la función vestibular angular. Clásicamente se utiliza para el canal horizontal, pero puede implementarse para evaluar los canales semicirculares anteriores y posteriores.

Objetivo: Explorar la sensibilidad y especificidad de esta prueba para los canales verticales a ojo desnudo en nuestro medio, en relación a la prueba calórica.

Material y método: Estudio prospectivo de evaluación de test diagnóstico. Se realizó prueba de impulso cefálico para todos los canales semicirculares a pacientes con indicación de evaluación funcional de VIII par.

Resultados: Se evaluaron 118 pacientes, donde 49,2\% presentó una prueba calórica clásica alterada. La sensibilidad del impulso cefálico para el canal posterior fue 13,1\% (y 3,2\% para el canal anterior) con una especificidad de 100\% para ambos. Para el canal horizontal la sensibilidad fue de 63,9\% y la especificidad de $100 \%$.

Discusión: La prueba de impulso cefálico para los canales anteriores y posteriores a ojo desnudo es altamente específico, pero muy poco sensible, teniendo una utilidad relativa dentro de la clínica, a diferencia de la misma prueba para el canal horizontal que con la misma especificidad pero con una sensibilidad aceptable representa un examen rápido y de simple ejecución.

Palabras clave: Reflejo óculo-vestibular, pruebas vestibulares funcionales.
\end{abstract}

\begin{abstract}
Introduction: The Head Impulse Head test represents a quick and simple technique for perioheral vestibular function assessment, by means of exploring the vestíbulo-ocular reflex. It is usually performed on the horizontal semicircular canals planes, but it can also explore the anterior and posterior canals.

Aim: To assess the head impulse test's sensitivity and specificity for the anterior and posterior canals on a bedside scenario, taking the classic caloric test as gold standard.

Material and method: Prospective test-assessment study. A head impulse test for every semicircular canal was performed on patients with indication of vestibular study with caloric test.
\end{abstract}

\footnotetext{
Médico. Departamento Otorrinolaringología, Pontificia Universidad Católica de Chile.

Tecnólogo Médico. Departamento Otorrinolaringología, Pontificia Universidad Católica de Chile.

Tecnólogo Médico. Centro Médico Alcántara.
} 
Results: 118 patients were evaluated, where 49,2\% presente an abnormal caloric test. The head impulse test's sensitivity for the posterior canal canal was $13,1 \%(3,2 \%$ for the anterior canal). The test's specificity was $100 \%$ for both vertical canals. On the horizontal plane, sensitivity reached $63,9 \%$, while specificity was $100 \%$

Discussion: The head impulse test for vertical (anterior and posterior) canal on a bedside scenario is highly specific but poorly sensitive, thus having a relatively low clinical utility. On the contrary the test for the horizontal canal remains a quick and simple tool, with acceptable sensitivy and great sensitivity for assessing vestibular function loss.

Key words: Vestibulo-ocular reflex, functional vestibular tests.

\section{INTRODUCCIÓN}

La patología del equilibrio y balance representa un motivo de consulta frecuente tanto en otorrinolaringología como en servicios de urgencia y atención en medicina general' ${ }^{1}$. Dentro de su estudio -y como consecuencia de importantes avances en la comprensión de la fisiología así como de la incorporación de nuevas tecnologíaslas pruebas de impulso cefálico, descritas hace décadas ${ }^{2-}$ ${ }^{4}$, han cobrado actualmente gran vigencia e importancia, tanto en la evaluación ambulatoria en el contexto de un examen físico otoneurológico básico ${ }^{5}$, como en el estudio diagnóstico definitivo de la función vestibular-9.

Estas pruebas se basan en la evaluación de la indemnidad del reflejo vestíbulo-ocular, al observar la capacidad de mantener la mirada en un objeto a pesar de movimientos rápidos de la cabeza provocados por el examinador (el detalle de la fisiología e interpretación de esta prueba diagnóstica puede encontrarse en un artículo previamente publicado en esta revista ${ }^{10}$.

En un paciente sano, el movimiento de los ojos ocurre con una latencia de 7 a 10 milisegundos (latencia imperceptible a ojo desnudo), en sentido perfectamente opuesto a la velocidad y eje de rotación de un movimiento cefálico. Como resultado se obtendrá la ilusión de que los ojos permanecen inmóviles con la vista fija en el objeto durante el examen (Figura 1).

En pacientes con patología esta respuesta se retrasa, siendo necesaria una sacada ocular correctiva (semejante a la fase rápida del nistagmo) para reposicionar el ojo hacia el objeto que se está mirando. Durante el examen se observará entonces que el ojo "sigue a la cabeza» desviando la mirada del objeto a un punto lejano. Un instante después (100 a 300 milisegundos), el ojo se moverá para redirigir la mirada hacia el objeto (sacada correctiva - Figura 2).

Este fenómeno es visible a ojo desnudo cuando la patología es severa, pero puede pasar inadvertido en situaciones leves y moderadas (cuando la sacada correctiva ocurre durante el movimiento de la cabeza y no después de éste) ${ }^{11}$. Es en estos últimos casos donde la incorporación de un acelerómetro para medir la velocidad del giro de la cabeza y una cámara de alta velocidad con la capacidad de una evaluación tridimensional del
Figura 1. Prueba de impulso cefálico normal.

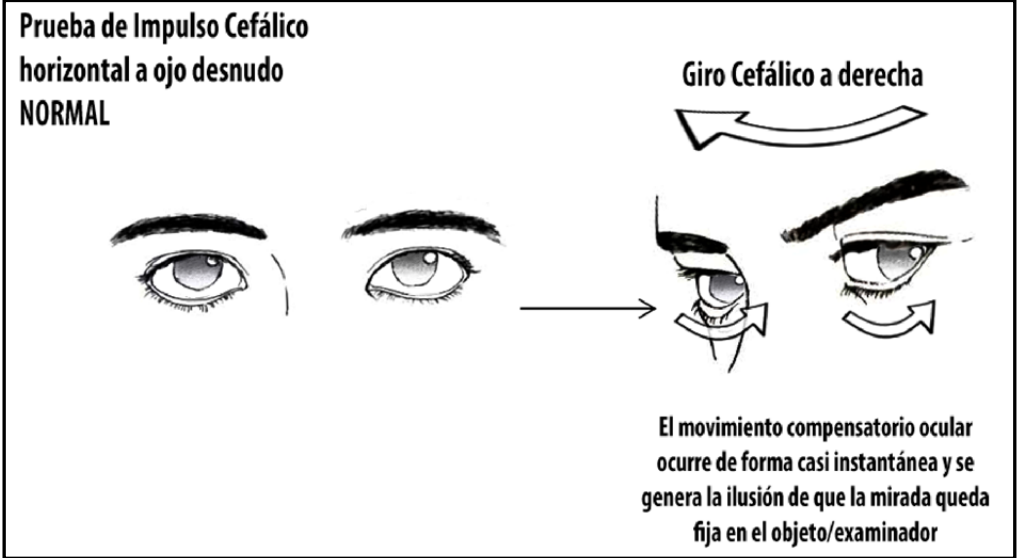




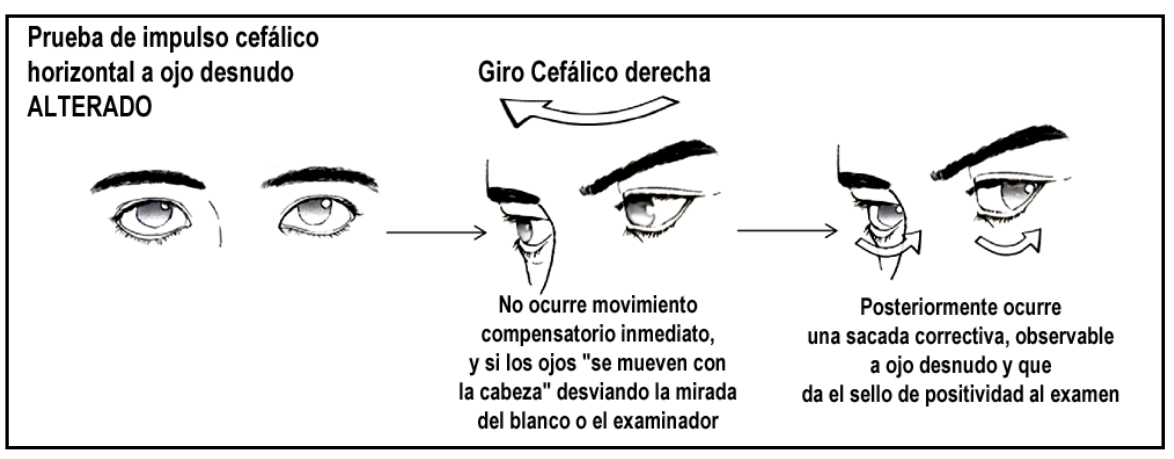

Figura 2. Prueba de impulso cefálico alterado. Sacada correctiva.

movimiento del globo ocular, ayuda a detectar el fenómeno con gran detalle y precisión ${ }^{7,8}$. Esta tecnología ya se encuentra disponible bajo el nombre de «video Head Impulse Test» (vHIT ), y será motivo de un próximo trabajo.

Para entender el real aporte y lugar de la prueba de impulso cefálico (HIT por sus siglas en inglés Head Impulse Test) dentro del contexto del estudio vestibular, conviene revisar el estado actual de la evaluación de la patología del equilibrio3,6,12.

Desde una perspectiva global es útil recordar al equilibrio como un sentido que resulta de la integración en el sistema nervioso central de los sistemas visual, propioceptivo y vestibular ${ }^{13}$. El examen de elección para evaluar esta tríada en su conjunto es la posturografía, y en muchos centros es considerado como la estrategia inicial al enfrentar al paciente con problemas de balance ${ }^{14}$.

De los tres, el sistema vestibular es el más sintomático cuando está alterado, especialmente cuando se asocia a cuadros vertiginosos, e históricamente ha concentrado la atención de la otorrinolaringología ${ }^{15}$. Desde un punto de vista funcional, podemos sistematizar su estudio en dos grandes ramas: una función angular (0 de canales semicirculares) y una función otolítica (o utrículosacular) $)^{6,16}$.

El estudio de la función otolítica puede a su vez dividirse en una evaluación de su componentes dinámicos (sensación de aceleración lineal) y estáticos (sensación de posición y orientación en reposo $)^{17}$. En lo dinámico, los potenciales miogénicos vestibulares (VEMPs por su sigla en inglés) serían el método de elección, donde los VEMPs oculares (oVEMP) evaluarían la función del utrículo, cuya información se conduce por el nervio vestibular superior (NVS), mientras que los VEMPs cervicales (cVEMP) explorarían al sáculo y el nervio vestibular inferior (NVI) ${ }^{18}$. Esta última característica (el poder evaluar la función del NVI) es una de las grandes fortalezas de los cVEMP. Internacionalmente se reconoce este examen como la primera opción para el estudio del NVI ${ }^{19}$.

La función otolítica estática por otro lado, es fácilmente evaluable a través de pruebas como el test subjetivo vertical u horizontal ${ }^{20}$.

Con respecto a la función angular -dependiente de los canales semicirculares- resulta útil separar al estudio del vértigo postural paroxístico benigno en un capítulo aparte, ya que su evaluación responde a una fisiopatología particular, ampliamente explorada a través de las diferentes maniobras de provocación postural existentes ${ }^{21}$.

Centrándonos en la función angular propiamente tal, la prueba calórica clásica ha sido históricamente el patrón de oro en su evaluación. Sin embargo es necesario recordar que esta prueba sólo explora la función del canal semicircular horizontal y el NVS que lo inerva ${ }^{6}$. Para estudiar el canal semicircular posterior y el NVI, existe la posibilidad de realizar la prueba calórica mínima22. Los estudios calóricos se basan en la estimulación del reflejo vestíbulo ocular, al generar un movimiento asimétrico (sólo se estimula un oído) de endolinfa al interior de un determinado canal, de baja velocidad angular (del orden de los 0,002 Hz) pero constante, utilizando como motor las diferencias de densidad en la endolinfa al interior del canal, inducidas por un estímulo térmico ${ }^{23}$. Otras opciones incluyen exámenes como la silla rotatoria, las que (sin depender de la indemnidad del oído medio y externo) generan un estímulo bilateral y prolongado de mediana velocidad angular (habitualmente entre $0,01 \mathrm{a} 0,8 \mathrm{~Hz}$, pero que puede llegar hasta $1,3 \mathrm{~Hz}$ ), cuyo análisis depende de varias subpruebas a distintas combinaciones de 
velocidades y formas de aceleración ${ }^{29}$. Este tipo de prueba sería más sensible que las pruebas calóricas (dado el mayor rango de velocidad angular de estimulación) pero mucho menos específica que ésta (en los rangos de aceleración y velocidad angular de la prueba, y dado que el estímulo es bilateral, es difícil distinguir una verdadera lesión vestibular de fenómenos normales de interacción entre ambos oídos estimulados, los que pueden «simular» una alteración del reflejo vestíbulo-ocular sin ser patológico) $)^{29,30}$.

Una nueva alternativa reside justamente en la prueba de impulso cefálico o HIT, la que explora el mismo reflejo vestíbulo-ocular que evalúa la prueba calórica, pero generando un movimiento de endolinfa al interior de una pareja de canales (un canal en cada oído), simétrico, breve y de alta velocidad angular (del orden de los $2 \mathrm{a} 5 \mathrm{~Hz}$ ), inducido por un movimiento cefálico producido por el examinador (emulando el estímulo fisiológico para el cual parecen estar diseñados los canales semicirculares) $)^{3,12}$.

Este tipo de examen presenta varias ventajas por sobre las pruebas calóricas tradicionales: no genera náuseas o síntomas vegetativos, toma escasos minutos en realizarse y puede evaluar la función vestibular independientemente de si existe patología 0 asimetría en la estructura del oído externo y medio (como una otitis media crónica), restricción que tienen las pruebas calóricas con agua ${ }^{3,9,10,23}$.

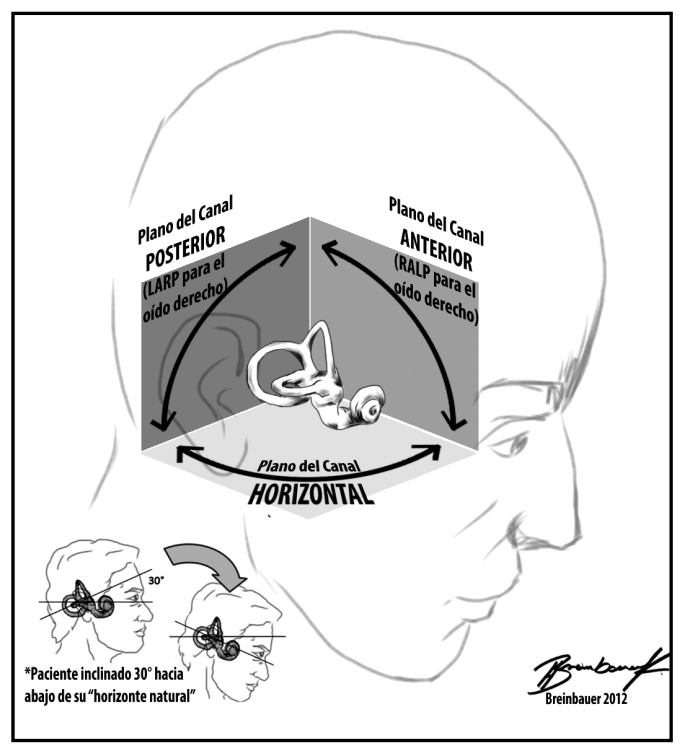

Figura 3. Disposición de canales semicirculares en el espacio. Visión lateral con cabeza inclinada en $30^{\circ}$.
El HIT representa entonces una alternativa sencilla y de rápida ejecución que evalúa aspectos similares a una prueba calórica, con la posibilidad de evaluar todos los canales semicirculares, aunque en la literatura internacional es el horizontal el canal más ampliamente estudiado, tanto por la facilidad de su ejecución como por su equivalencia conceptual al estudio con la prueba calórica 3,4,8-10,24.

Para realizar una prueba de impulso cefálico para los canales verticales (anterior y posterior) es necesario recordar la anatomía de los canales semicirculares, los que se organizan en una disposición prácticamente ortogonal, generando tres planos que cubren las tres dimensiones del espacio (Figuras 3 y 4). Estos planos son el Horizontal (que alinea los canales laterales de ambos oídos), el RALP (Right Anterior- Left Posterior, que alinea el canal Anterior del oído derecho con el canal posterior del oído izquierdo) y el LARP (Left Anterior - Right Posterior, que alinea el canal anterior izquierdo y el posterior derecho).

Para realizar el examen en los ejes RALP o LARP, una posibilidad es -siempre con la cabeza inclinada $30^{\circ}$ hacia adelante llevando los canales horizontales al «horizonte verdadero»- girar la cabeza hacia un lado en $45^{\circ}$ (para el eje RALP, se giraría hacia la izquierda del paciente - Figura 5). En esta posición, los canales anterior derecho y posterior izquierdo estarían alineados en un eje vertical

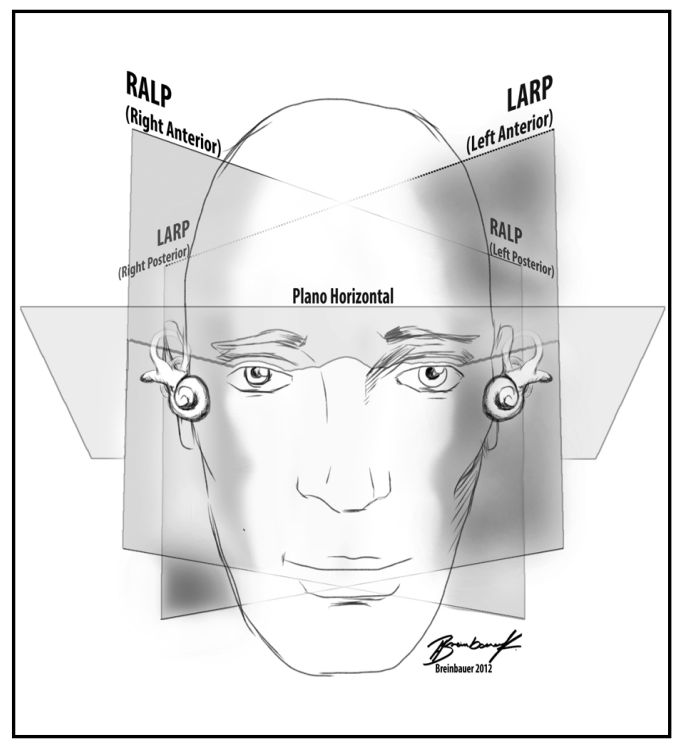

Figura 4. Planos Horizontal, RALP y LARP. 


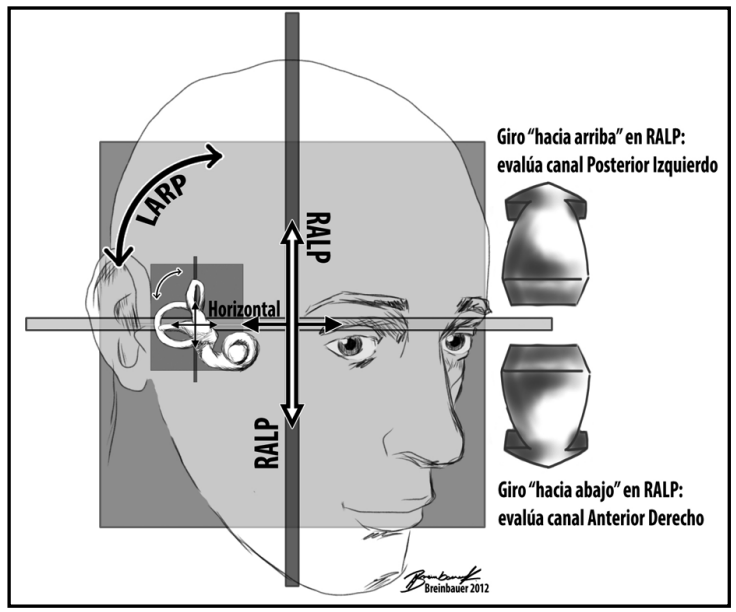

Figura 5. Interpretación de la prueba de impulso cefálico en plano RALP para el canal anterior derecho y posterior izquierdo.

con un examinador posicionado al frente del paciente $e^{2,25}$.

Dado que la estimulación de los canales anteriores y posteriores ocurre con corrientes de endolinfa ampulofugas (que se alejan de las crestas ampulares), un movimiento en el plano descrito hacia abajo genera un estímulo del canal anterior correspondiente (derecho en RALP) y una inhibición del canal posterior correspondiente (izquierdo en LARP) ${ }^{26}$. Lo contrario ocurre con un giro de la cabeza hacia arriba, con una estimulación del canal posterior y una inhibición del canal anterior correspondiente (Figura 5).

A la velocidad angular a la que se generan impulsos cefálicos (sobre los $2 \mathrm{~Hz}$ ), la información inhibitoria se satura muy rápidamente (dado que el rango dinámico desde el tono vestibular basal en reposo hasta la mínima inhibición es muchísimo más estrecho que el rango desde el tono basal hasta la excitación producida por un impulso cefálico), por lo que aunque un giro siempre genere un movimiento de endolinfa en los dos canales semicirculares que determinen uno de los planos (Horizontal, RALP o LARP), la interpretación del fenómeno siempre corresponderá prioritariamente al canal semicircular que reciba un estímulo excitatorio ${ }^{27}$ (esto no ocurre cuando la velocidad angular del estímulo es menor, como en la silla rotatoria, en cuyo caso la información inhibitoria -la que no se satura- es equivalente en magnitud a la excitatoria y resulta inapropiado interpretar el resultado de forma unilate$\mathrm{ral}^{27-30}$ ). Esto permite una interpretación unilateral del examen: movimientos hacia abajo en la posición des- crita previamente evalúan la función de los canales anteriores, y los movimientos hacia arriba, la función de los posteriores.

\section{OBJETIVO}

El objetivo del presente trabajo es explorar la sensibilidad, especificidad y características de la prueba de impulso cefálico para los canales verticales a ojo desnudo, utilizando la prueba calórica como referente de la función vestibular.

\section{MATERIAL Y MÉTODO}

Estudio prospectivo de evaluación de test diagnóstico, realizado entre abril y julio de 2012 en la Red de Salud UC.

Considerando una potencia estadística de $80 \%$, un error alfa de $5 \%$ y en base a resultados de un estudio previo por los mismos autores ${ }^{10}$, se calculó un tamaño muestral necesario de 58 pacientes para encontrar valores confiables de sensibilidad y especificidad.

La muestra se escogió por criterios de factibilidad (no probabilística) y consistió en pacientes a quienes se les hubiese indicado la realización de un Estudio Funcional de VIII par dentro de la Red de Salud UC. Se consideraron como criterios de exclusión la presencia de nistagmo espontáneo al momento de la realización del estudio vestibular, así como contar con historia de patología de equilibrio conocida previamente.

Se realizó adiestramiento de los evaluadores (tres tecnólogos médicos) en la realización de la prueba de impulso cefálico tanto para los canales horizontales como verticales, siguiendo la descripción revisada en la introducción y en el estudio previo de los autores para el canal horizontal exclusivo ${ }^{10,26}$.

Previo a la realización de la prueba calórica durante el estudio de VIII par, se realizó la prueba de impulso cefálico para los tres planos (horizontal, RALP, LARP) en sus dos sentidos (izquierda-derecha, abajo-arriba) registrando la positividad del examen como una pérdida de la fijación de la mirada continua en el evaluador, con la aparición de una sacada de corrección.

Se realizaron cálculos de sensibilidad y especificidad, utilizando los resultados de la prueba calórica clásica como patrón de oro. 
En nuestro centro la prueba calórica mínima representa una posibilidad de estudio de la función angular del canal semicircular posterior, sin embargo durante el periodo estudiado se realizaron sólo 8 Pruebas Calóricas Mínimas que mostraron alteraciones y que no cumplieron los criterios de exclusión, por lo que no se incorporaron dentro de los análisis formales y sólo se revisará de forma descriptiva en los resultados.

El análisis estadístico se llevó a cabo con Software estadístico SPSS 20.

\section{RESULTADOS}

Se evaluaron 118 pacientes, $61 \%$ de los cuales fueron mujeres con una edad promedio de 51,2 años (desviación estándar 18,9 años). En 57 casos la prueba calórica resultó normal. Dentro de este grupo la prueba de impulso cefálico resultó normal en todos los casos, para todos los canales.

Dentro de los 61 pacientes con prueba calórica alterada, 39 pacientes presentaron un HIT alterado para canales horizontales, 8 pacientes presentaron alteración en la prueba para el canal posterior (que también mostraban alteración ipsilateral para el canal lateral) y sólo 2 presentaron alteración para el canal anterior (estos últimos dos pacientes también mostraban alteraciones ipsilaterales para los canales posterior y horizontal).

En la Tabla 1 podemos apreciar los valores de sensibilidad y especificidad del HIT para cada canal en particular, considerando el resultado de la prueba calórica clásica como patrón de oro.
Destaca el $100 \%$ de especificidad de la prueba en cualquier escenario. Sin embargo, y aunque la sensibilidad para el canal horizontal $(63 \%$ en estudio) es aceptable y dentro de los rangos previamente descritos ${ }^{10,28}$, la sensibilidad de la prueba para el canal posterior $(13,1 \%)$ es bastante baja, y aún peor para el canal anterior (3,2\%).

En cuanto considerar otra prueba que evalúe algún canal vertical como patrón de referencia, se intentó evaluar la relación del HIT con la prueba calórica mínima. Sin embargo se realizaron sólo 13 pruebas calóricas mínimas durante el estudio, 8 de las cuales presentaron alteración. Dentro de estas últimas, 2 presentaron una alteración del HIT para el canal posterior. Dado el bajo número de casos se prefirió no realizar mayor análisis estadístico.

\section{DISCUSIÓN}

En nuestro estudio las pruebas de impulso cefálico para los canales verticales resultaron ser altamente específicas pero muy poco sensibles, con valores bajo el $13 \%$, lo que le otorga una baja utilidad clínica práctica, muy en contraste con la versión de la prueba para los canales horizontales.

Creemos que la explicación más plausible para esta baja sensibilidad de la prueba en los canales verticales se relaciona con la dificultad de realizar el giro cefálico en los planos RALP y LARP.

En términos prácticos tanto para el paciente como para el examinador es mucho más fácil ejecutar la maniobra en un sentido horizontal, desde una

Tabla 1. Prueba de impulso cefálico para cada tipo de canal semicircular en comparación a prueba calórica

\begin{tabular}{|c|c|c|c|c|c|c|c|}
\hline & & $\begin{array}{l}\text { Prueba } \\
\text { calórica } \\
\text { alterada }\end{array}$ & $\begin{array}{l}\text { Prueba } \\
\text { calórica } \\
\text { Normal }\end{array}$ & Sensibilidad & Especificidad & $\begin{array}{c}\text { Valor } \\
\text { Predictivo } \\
\text { Positivo }\end{array}$ & $\begin{array}{c}\text { Valor } \\
\text { Predictivc } \\
\text { Negativo }\end{array}$ \\
\hline \multicolumn{8}{|c|}{ Prueba de impulso cefálico } \\
\hline Canal & Alterado & 39 & 0 & $63,9 \%$ & $100 \%$ & $100 \%$ & $72,1 \%$ \\
\hline Horizontal & Normal & 22 & 57 & & & & \\
\hline Canal & Alterado & 8 & 0 & $13,1 \%$ & $100 \%$ & $100 \%$ & $51,8 \%$ \\
\hline Posterior & Normal & 53 & 57 & & & & \\
\hline Canal & Alterado & 2 & 0 & $3,2 \%$ & $100 \%$ & $100 \%$ & $49,1 \%$ \\
\hline Anterior & Normal & 59 & 57 & & & & \\
\hline Total ${ }^{*}:$ & & 61 & 57 & & & & \\
\hline
\end{tabular}

* Total en relación a tipo resultado de prueba calórica, y es igual para la evaluación de cada canal. Cada canal se trató como una prueba independiente.

** Todos los pacientes con alteración en el canal posterior, presentaron alteración en el canal horizontal.

*** Todos los pacientes con alteración en el canal anterior, presentaron alteración tanto en el canal posterior como en el canal horizontal. 
posición inicial en que ambos están frente a frente, que en sentido vertical, donde la posición inicial (con la cabeza del paciente comienza orientada $45^{\circ}$ grados hacia un lado, mirando un poco «de reojo» al examinador) ya es de por sí algo incómoda.

En experiencias internacionales, tanto la amplitud como la velocidad lograda al realizar un HIT en canales verticales son significativamente menores que en el plano horizontal ${ }^{26}$. Existen otras técnicas para realizar el impulso en los ejes RALP y LARP, que consisten en movimientos diagonales, combinando equilibradamente un movimiento de torsión e inclinación en un eje antero-posterior y laterolateral, evitando un movimiento con un eje superoinferior (giro horizontal) ${ }^{2,25}$. Esta alternativa en la técnica del giro cefálico puede ser más precisa en un escenario de laboratorio con la retroalimentación de un equipo que indica los grados de torsión en cada plano utilizando un acelerómetro adosado a la cabeza del paciente. Sin embargo parece poco práctica en la clínica, y los autores reconocen en su experiencia el difícil entrenamiento del evaluador ${ }^{2}$.

Pese a esto, no creemos que el reflejo vestíbuloocular no sea evaluable para los canales verticales, sino sólo que es más difícil y quizás no implementable en un contexto ambulatorio, sino con el apoyo de equipo apropiado. Utilizando la técnica alternativa que acabamos de describir, pero sobre todo utilizando métodos de registro que permitan seguir con precisión tanto el movimiento de la cabeza (acelerómetro) y de los ojos (bobina escleral2, o más recientemente video-oculografía de alta velocidad con reconstrucción tridimensional ${ }^{26}$ ha sido posible identificar no solo la función de cada canal de forma individual, sino pacientes con hipofunción específica (y exclusiva) de cada uno ${ }^{25}$.

Quizás teniendo que abandonar la idea de contar con una prueba «al lado de la cama del enfermo» para los canales verticales, no debemos olvidar el importante rol de la prueba de impulso cefálico para los canales horizontales usada a ojo desnudo (hHIT). Esta herramienta puede dar, en más de la mitad de las veces, una información equivalente a una alteración encontrada en una prueba calórica clásica, y prácticamente sin riesgo de falsos positivos. Es importante recalcar que se puede realizar incluso con la presencia de nistagmo espontáneo, lo que suma un factor importante al intentar aumentar la certeza de un diagnóstico otoneurológico, solo con un examen físico sistemático.
El estudio de Newman-Toker en $2008^{5}$ es quizás uno de los que más realza la utilidad clínica del HIT horizontal a ojo desnudo. En este trabajo se encontró que aunque tanto pacientes con patología central como periférica podían presentar un HIT horizontal alterado, un HIT horizontal normal en el contexto de un paciente con un síndrome vertiginoso (con o sin nistagmo) se relacionaba en $91 \%$ con patología central.

\section{CONCLUSIONES}

En síntesis, la prueba de impulso cefálico realizada a ojo desnudo para el canal horizontal representa una poderosa herramienta dentro de la evaluación del paciente con trastorno de equilibrio y pudiese ser implementada de forma regular en la práctica clínica cotidiana, mientras que su versión para los canales verticales no tendría mayor relevancia dada una muy baja sensibilidad.

Queda por ver si con el apoyo de nueva tecnología como el vHIT, las pruebas de impulso cefálico podrían convertirse en un complemento crucial 0 una alternativa razonable a las pruebas calóricas en el estudio del componente angular de la función angular para todos los canales semicirculares.

\section{BIBLIOGRAFÍA}

1. Yin M, Ishikawa K, Wong W, Shibata Y. A clinical epidemiologial study in 2169 patients with vertigo. Auris Nasus Larynx 2009; 36: 30-5.

2. Cremer P, Halmagyi G, Aw S, et al. Semicircular canal plane head impulses detect absent function of individual semicircular canals. Brain 1998; 121: 699-716.

3. Halmagy G, Curthoys I. A clinical sign of canal paresis. Arch Neurol 1988; 45: 737-9.

4. Halmagy G, Curthoys I, Cremer P, et al. The human horizontal vestibulo-ocular reflex to high acceleration stimulation before and after unilateral vestibular neurectomy. Exp Brain Res 1990; 81: 479-90.

5. Newman-Toker D, Kattah J, Alvernia J, Wang D. Normal head impulse test differentiates acute cerebellar strokes from vestibular neuritis. Neurology 2008; 70(24): 2378-85.

6. CuRthors I. The interpretation of clinical tests of peripheral vestibular function. Laryngoscope 2012; 122(6): 1342-52. 
7. Barti K, Lehnen N, Kohlbecher S, y C. Head impulse testing using video-oculography. Ann N Y Acad Sci 2009; 1164: 331-3.

8. MacDougall $\mathrm{H}$, Weber $\mathrm{K}, \mathrm{LA} \mathrm{M}, \mathrm{y}$ c. The video head impulse test: diagnostic accuracy in peripheral vestibulopathy. Neurology 2009; 73(14): 1134-41.

9. Weber K, MacDougall H, Halmagy $G$, y c. Impulsive testing of semicircular-canal function using video-oculography. Ann N Y Acad Sci 2009; 1164: 486-91.

10. Breinbauer $\mathrm{H}$, Anabalon J. Prueba de impulso cefálico. Rev Otorrinolaringol Cir Cabeza Cuello 2011; 71: 123-30.

11. Tuernström F, Nyström A, Magnusson M. How to Uncover the Covert Saccade During the Head Impulse Test. Otol Neuroto/2012; 33(9): 1583-5.

12. Wuyts F, Furman J, Vanspaumen R, Vander Heyning P. Vestibular function testing. Curr Opin Neurol 2007; 20(1): 19-24.

13. Visser J, Carpenter M, van der Koolj H, Vloem B. The clinical utility of posturography. Clin Neurophysiol 2008; 119(11): 2424-36.

14. Zhang D, Fan Z, Yu G. The clinical value of dynamic posturography in the peripherical vertigo. Nin Chung Er Bi Yan HouTou Jing WaiKeZaZhi2009; 23(16): 721-3.

15. Lal Y, Wang T, Chuang L, Chen M, Wang P. Epidemiology of Vertigo: A National Survey. Otolaryngol Head Neck Surg 2011; 145(1): 110-6.

16. UCHINO Y, KUSHIRO K. Differences between otolith- and semicircular canal-activated neural circuitry in the vestibular system. Nerosci Res 2011; 71: 315-27.

17. CuRTHOYs I. A critical review of the neurophysiological evidence underlying clinical vestibular testing using sound, vibration and galvanic stimuli. Clin Neurophysiol 2010; 121: 132-44.

18. Brantberg K. Vestibular evoked myogenic potentials (VEMPs): usefulness in clinical neurotology. Semin Neurol 2009; 29: 541-7.

19. Rosengren S, Welgampola M, Clobatch J. Vestibular evoked myogenic potentials: past, present and future. Clin Neurophysiol 2010; 121: 636-51.

20. Halmagy G, Curthoys I. Clinical testing of otolith function. Ann N Y Acad Sci 1999; 871: 195-204.

21. Bhattacharyya N, Baugh R, Orvidas L, Barrs $D$, Bronston L, CASS S, et AL. Clinical practice guideline: benign paroxysmal positional vertigo. Otolaryngol Head Neck Surg 2008; 139(5 Suppl 4): S47-81.

22. Riveros H, Callejas C, Nieme C, Gajardo P, Anabalón J, Correa C. Importancia clínica de la prueba calórica mínima. Rev Otorrinolaringol Cir Cabeza Cuello 2007; 67: 217-21.

23. Zapala D, OLSholt K, Lundy L. A comparison of water and air caloric responses and their ability to distinguish between patients with normal and impaired ears. Ear Hear 2008; 29: 585-90.

24. WeBer K, Aw S, Todd M, Y c. Horizontal impulse test detects gentamicin vestibulotoxicity. Neurology 2009; 72(16): 1417-24.

25. Aw S, Fetter M, Cremer P, Karlberg M, Halmagy G. Individual semicircular canal function in superior and inferior vestibular neuritis. FALTA NOMBRE DE REVISTA 57. 2001: 768-74.

26. Ulmer E, Bernard-Demanze L, Lacour M. Statistical study of normal canal deficit variation range. Measuremet using Head impulse Test video system. Eur Ann Otorhinolaryngol Head Neck Dis 2011; 128: 278-82.

27. Strupp M, Brandt T. Vestibular Neuritis. Semin Neurol 2009; 29: 509-19.

28. Pérez N, Rama-López J. Head-Impulse and Caloric Tests in Patients with Dizziness. Otol Neurotol 2003; 24(6): 913-7.

29. Palomar-Asenjo V, Boleas Aguirre MS, SánchezFerRándiz N, Pérez N. Caloric and Rotatory Chair Test Results in Patients with Ménière's Disease. Otol Neurotol 2006; 27: 945-50.

30. Arriaga MA, Chen DA, Cenci KA. Rotational chair (ROTO) instead of electronystagmography (ENG) as the primary vestibular test). Otolaryngol Head Neck Surg 2005; 133(3): 329-33.

Dirección: Hayo Breinbauer K. 